\title{
Targeted Macromolecules Delivery by Large Lipidic Nanovesicles Electrofusion with Mammalian Cells
}

\author{
Pascal Demange ${ }^{1,2}$, Valérie Réat ${ }^{1,2}$, Stefan Weinandy ${ }^{1,2}$, Remy Ospital ${ }^{1,2}$, Louise Chopinet-Mayeux ${ }^{1,2}$, \\ Pauline Henri ${ }^{1,2}$, Alain Milon ${ }^{1,2}$, Justin Teissié ${ }^{1,2^{*}}$ \\ ${ }^{1}$ Centre National de la Recherche Scientifique (CNRS), Institut de Pharmacologie et de Biologie Structurale (IPBS), Toulouse, \\ France; ${ }^{2}$ Université de Toulouse, Université Paul Sabatier (UPS), IPBS, Toulouse, France. \\ E-mail: *Justin.teissie@ipbs.fr
}

Received October $3^{\text {rd }}, 2011$; revised November $4^{\text {th }}, 2011$; accepted November $14^{\text {th }}, 2011$.

\begin{abstract}
Lipidic nanovesicles (so called liposomes) were one the earliest forms of nanovectors. One of their limits was our lack of knowledge on the delivery pathway of their content to the target cell cytoplasm. The present communication describes an efficient way to enhance the delivery. Pulsed electric fields (PEF) are known since the early 80's to mediate a fusogenic state of plasma membranes when applied to a cell suspension or a tissue. Polykaryons are detected when PEF are applied on cells in contact during or after the pulses. Heterofusion can be obtained when a cell mixture is pulsed. When lipidic nanovesicles, either small unilamellar vesicles (SUVS) or large unilamellar vesicles (LUVS), are electrostatically brought in contact with electropermeabilized cells by a salt bridge, their content is delivered into the cytoplasm in electropermeabilized cells. The PEF parameters are selected to affect specifically the cells leaving the vesicles unaffected. It is the electropermeabilized state of the cell membrane that is the trigger of the merging between the plasma membrane and the lipid bilayer. The present investigation shows that the transfer of macromolecules can be obtained; i.e. $20 \mathrm{kD}$ dextrans can be easily transferred while a direct transfer does not take place under the same electrical parameters. Cell viability was not affected by the treatment. As delivery is present only on electropermeabilized cells, a targeting of the effect is obtained in the volume where the PEF parameters are over the critical value for electropermeabilization. A homogeneous cytoplasm labeling is observed under digitised videomicroscopy. The process is a content and "membrane" mixing, following neither a kiss and run or an endocytotic pathway.
\end{abstract}

Keywords: Electrofusion, Delivery Systems in Cancer, Liposomes, Lipidic Nanovesicles

\section{Introduction}

New methods for large molecules delivery in cells remain a major problem in pharmacology. The main problem is to provide a pathway across the cell membrane with no effect on the cell viability. Using liposomes (the magic bullet) to target the delivery was a promising approach but it faces the limit of a poor transfer. Pulsed electric fields (PEF) are known since the early 80's to mediate a fusogenic state of plasma membranes when applied to a cell suspension or a tissue [1-3]. Polykaryons are detected when PEF are applied on cells in contact during or after the pulses [4]. Heterofusion can be obtained when a cell mixture is pulsed. But the two partners should have a similar size if the mixture is pulsed due to the fact that the PEF effect is strongly size dependent.
Nevertheless we reported that when lipidic nanovesicles (SUVs or LUVs) are electrostatically brought in contact with electropermeabilized cells by a salt bridge, their content was delivered in the cytoplasm [5]. This was experimentally directly detected by the transfer of a small soluble fluorescent dye, Pyranin, trapped in the nanovesicles to the cell cytoplasm. A homogeneous cytoplasm labeling is observed under digitized videomicroscopy. The PEF parameters were adjusted to affect specifically the cells leaving the vesicles unaffected by a direct effect of the PEF. Under these specific experimental conditions, the nanovesicles were too small to be affected by the field and thus only the electropermeabilized state of the cell membrane was the trigger of the merging between the plasma membrane and the lipid bilayer. No 
deleterious by-effect of PEF was detected on the cell viability. The biophysical processes supporting the merging between intact nanovesicles and electropermeabilized (fusogenic) cells is still under investigation. Membrane fusion is a key process in life. It occurs when two membranes, brought in close contact, merge in a single one. A close approach of two membranes (as needed for fusion to occur) is prevented by repulsive forces [6-8]. In the case of synaptic vesicles, SNAREs bring the contact but the following steps where a transient $\mathrm{Ca}^{2+}$ interfacial increase occurs, are unclear $[9,10]$. The means, by which the repulsive forces between the two partners are overcome, remain poorly understood. But clearly a membrane destabilization should occur [11]. It may result from structural alterations of the target (plasma) membrane under a transient electric stress [12].

Taking into account, on one hand that membranes are electrically charged interfaces and on the other hand that electrical modulations bring cell membranes to a destabilized but spontaneously competent for fusion state, the hypothesis of Rosenheck [13] that a rate limiting step in membrane coalescence is due to the contact of electrically destabilized membranes with small vesicles, can be relevant of our observation. The model of Rosenheck was recently extended to vesicles [14]. If electrofusion between cells and lipid vesicles obeys the Rosenheck's model, then macromolecules transfer should be obtained as in the case of exocytosis. This was checked in the present study. Due to their large inner volume, large unilamellar vesicles (LUV) were selected as nanovesicles to give a significant delivery to the cytoplasm. Fluorescent dextrans were selected to monitor the resulting transfer by digitized fluorescent microscopy and to valid the proof of concept.

\section{Materials and Methods}

\subsection{Cells}

The wild type Toronto cells (WTT), is derived from Chinese hamster ovary cells (CHOs) and was first introduced in the 1960s. It can grow in suspension and in culture flasks (generation time $=18-20$ hours).

The property to grow in suspension prevents the necessity of trypsinisation.

MEM 0111 buffer (Eurobio France (ref: CM1MEM40K-BP)) with Foetal Calf Serum 8\%, (SVF EUROBIO, ref: CVFSVF00-01, lot no: S155839), D (+) - Glucose $45 \%$, (3.5 g/l) (Sigma, USA), Tryptose phosphate (2.95 g/l), Vitamins (GIBCO, ref: 043-01040) and Antibiotics (penicillin 100 units $/ \mathrm{ml}$, streptomycin $100 \mathrm{mg} / \mathrm{ml}$, Lglutamin $0.58 \mathrm{mg} / \mathrm{ml}$ ) is used to cultivate the $\mathrm{CHO}$ cells under slow agitation $\left(70\right.$ to $100 \mathrm{rpm}, 37^{\circ} \mathrm{C}$ ). Cells stay in the exponential growth phase by a control of the cell number (dilution from 0.55 up to $0.7 \times 10^{6}$ cells every day).

\subsection{Vesicles}

The lipids were Cholesterol (362794, SIGMA), L- $\alpha-$ Phosphatidylserine (840032, AVANTI), 1,2-Dioleoyl-snGlycero-3-Phosphocholine (DOPC, 850375, AVANTI).

$8.5 \mathrm{mg}$ of PC/PS/Cholesterol lipid mixture (6/1/3 molar ratio) was dissolved in chloroform. Lipids were dried under nitrogen flow and vacuum $(30 \mathrm{~min})$. They were then resuspended in $5 \mathrm{mM}$ Hepes $(\mathrm{pH}$ 7.2) containing Fluorescein labeled $20 \mathrm{kD}$ dextrans (labeled-FD20) and vortexed to form large multilamellar vesicles (LMV). The temperature of HEPES buffer was kept above gelliquid crystal transition temperature of the lipid mixture (i.e. $-20^{\circ} \mathrm{C}$ for DOPC). The suspension was sonified (3 times 5 minutes, with 3 min break between each treatment) to obtain smaller unilamellar vesicles (SUV), then centrifuged at $13,000 \mathrm{~g}$ to remove of the titanium debris which came from the sonication tip. A 5 times freezing/thawing process followed to form bigger vesicles. Large unilamellar vesicles were obtained by extrusion through $0.2 \mu \mathrm{m}$ pore polycarbonate membrane (The Mini-Extruder (AVANTI)). The size homogeneity was checked by dynamic light scattering (Proteinsolution Dynapro)

\subsection{Electropulsation}

Cells were suspended in a $10 \mathrm{mM}$ HEPES buffer $(\mathrm{pH}$ 7.2), $250 \mathrm{mM}$ sucrose and $5 \mathrm{mM} \mathrm{CaCl} 2$ at a high cell density $\left(4 \times 10^{7} \mathrm{c} / \mathrm{ml}\right)$. The "pulsing" buffer contained $\mathrm{CaCl}_{2}$ to ensure the connection between cells and liposomes via electrostatic interaction. Low conductivity was important to decrease the Joule effect. A unipolar $G H T$ 1287 generator (Jouan, France) was used to provide rectangular pulses as high as $1 \mathrm{kV}$ with a constant intensity over the $100 \mu$ s pulse duration. An oscilloscope (Metrix OX 520 B, France) ensured the monitoring of the pulse. The generator was connected to two flat parallel stainless steel electrodes with an inter-electrode distance of $4 \mathrm{~mm}$. The edges of the electrodes were in contact with the bottom of a plastic Petri dish $($ Nunc $35 \times 10)$ to build an open pulsing chamber. Cells $\left(0.1 \mathrm{ml}\right.$ at $\left.4 \times 10^{7} \mathrm{c} / \mathrm{ml}\right)$ were put between the two electrodes, together with a labelled-Dextran (FD20) loaded LUV suspension and/or HEPES buffer. 10 or more pulses $(480 \mathrm{~V}=1.2 \mathrm{kV} / \mathrm{cm}$, $100 \mu$ s, frequency $1 \mathrm{~Hz}$ ) were applied. After pulse delivery cells were kept 10 minutes at $25^{\circ} \mathrm{C}$.

A "washing" PBS buffer (Dulbecco's phosphate buffered saline, Eurobio, France, $\mathrm{pH} 7.2,-\mathrm{Ca}^{2+},-\mathrm{Mg}^{2+}$ ) was added. It contained phosphate ions to chelate calcium and remove the electrostatic bridges. The unfusioned nanovesicles were not bound anymore to the surface of the 
cells and could be washed out by centrifugation $(5 \mathrm{~min}$, $100 \times \mathrm{g})$.

The cell pellet was resuspended once again in PBS and analysed.

\subsection{Analysis}

Cells were analysed with the fluorescence digitized microscope Leica DM IRB (Wetzlar, Germany) using the filter set for fluorescein. Video monitoring was possible with a cooled CCD camera (Princeton Instruments, NJ, USA). The pictures were taken with the Metavue software (Molecular Devices, USA). Images were analysed with ImageJ (for more information http://rsb.info.nih.gov /ij/index.html). No filtering was operated on the raw data. A ROI was selected and was analysed by the "surface plot" operation.

\section{Results}

\subsection{LUV Characterization}

Fluorescein labeled $20 \mathrm{kD}$ dextrans were used to make sure that the electric treatment was not just inducing an exchange (by a "kiss and run" process) of small molecules as previously shown with Pyranin [5].

To eliminate the labeled $20 \mathrm{kD}$ dextrans that was not trapped in the vesicles, the extrusion method was followed by a G200 chromatography. The elution profiles are shown in Figure 1. LUVs were in the excluded fraction volume (fraction 10 to 15 ). Strong fluorescein absorption was detected on a broad range of fractions, starting from fraction 16. It was due to the elution of free labeled $20 \mathrm{kD}$ dextrans (untrapped in vesicles).

Furthermore, one single maximum in fluorescein absorption was detected suggesting homogeneity of the fluorescent specie. This was indicative that no free fluorescein (without dextran, i.e. with a low molecular weight) was trapped in the LUVs.

LUVs were used after the gel chromatography.

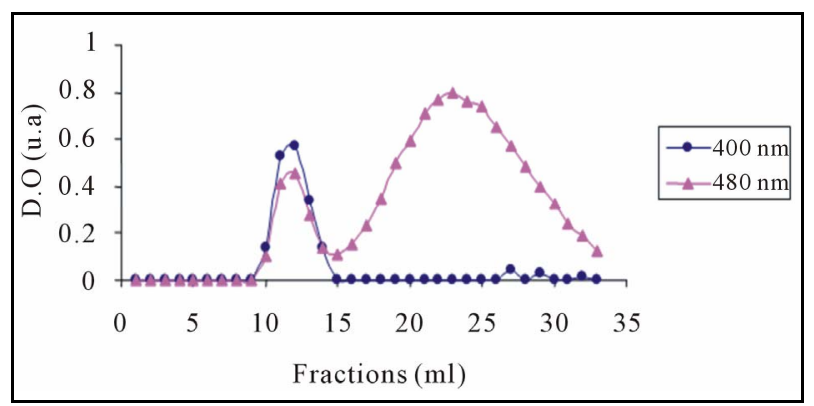

Figure 1. Elution profiles at 400 and $480 \mathrm{~nm}$ Extruded LUV solutions were analysed by gel chromatography. "Absorption" at $400 \mathrm{~nm}$ is due to the scattering of light by LUVs. Absorption at $480 \mathrm{~nm}$ is due to fluorescein bound to dextrans.

\subsection{Fusion}

Labeled $20 \mathrm{kD}$ dextrans could not be directly electroloaded in $\mathrm{CHO}$ cells under the electro pulsing conditions of $10 \times 0.1 \mathrm{~ms}$ [15].

The cells and LUVs $(3 / 1, \mathrm{~V} / \mathrm{V})$ were premixed in HEPES $10 \mathrm{mM}$, pH 7.2, sucrose $0.25 \mathrm{M}, \mathrm{CaCl}_{2} 5 \mathrm{mM}$ ("pulsing" buffer). An electrostatic bridge bound the negatively charged liposomes to the cells. No pulse was applied. After $10 \mathrm{~min}$ incubation, the mixture was washed in PBS buffer to chelate $\mathrm{Ca}^{2+}$ ions and remove the free liposomes. Cells were then incubated at $37^{\circ} \mathrm{C}$ up to 4 hours. As shown in Figure 2, no evidence of fusion was obtained as no fluorescence could be detected in the cytoplasm. No endocytotic process was indeed detected as no fluorescent spot could be detected even with the very sensitive detection obtained with the cooled CCD camera and the image processing. In order to get electrofusion, a similar protocol was followed but 10 or 20 square pulses $(1.2 \mathrm{kV} / \mathrm{cm}, 0.1 \mathrm{~ms}, 1 \mathrm{~Hz})$ were applied on the cell/LUVs mixture in the "pulsing" buffer. Fusion was then detected by the content mixing making the cell cytoplasm fluorescent as observed under a digitized fluorescent microscope (Figure 3).

For both experiments, fluorescence was present in a rather homogeneous pattern (an egg shell surface profile was observed). The roughness of the distribution (Figures 3(a) and 3(b) surface plot) can be just relevant of the noise of the signal as the magnitude of the "hot" spots

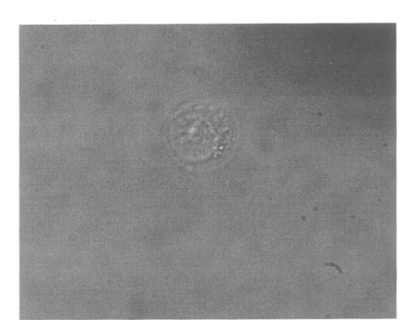

Contrast

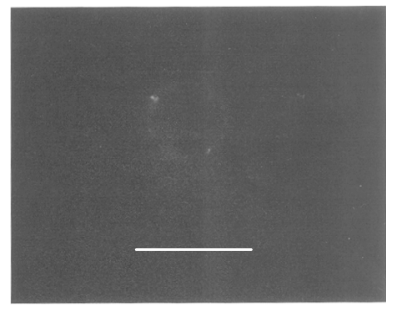

Fluorescence

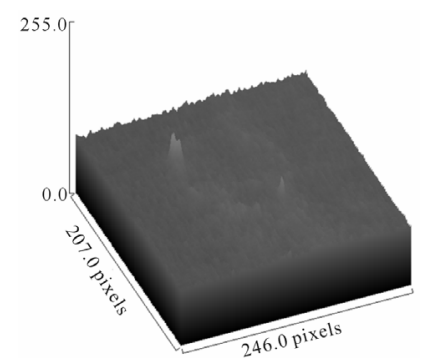

Figure 2. No spontaneous fusion is present between cells and LUVs Cells and LUVs were just mixed in the pulsing buffer. No fusion with LUVs was observed without electropermeabilization as no fluorescence was detected in the cytoplasm as shown on the right by the surface plot diagram. The bar is $20 \mu \mathrm{m}$ long. 


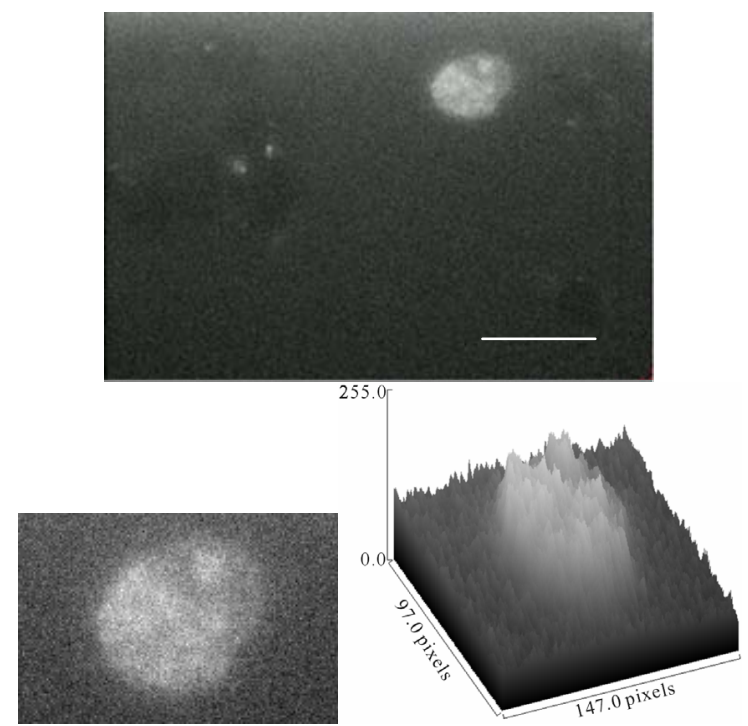

(a)

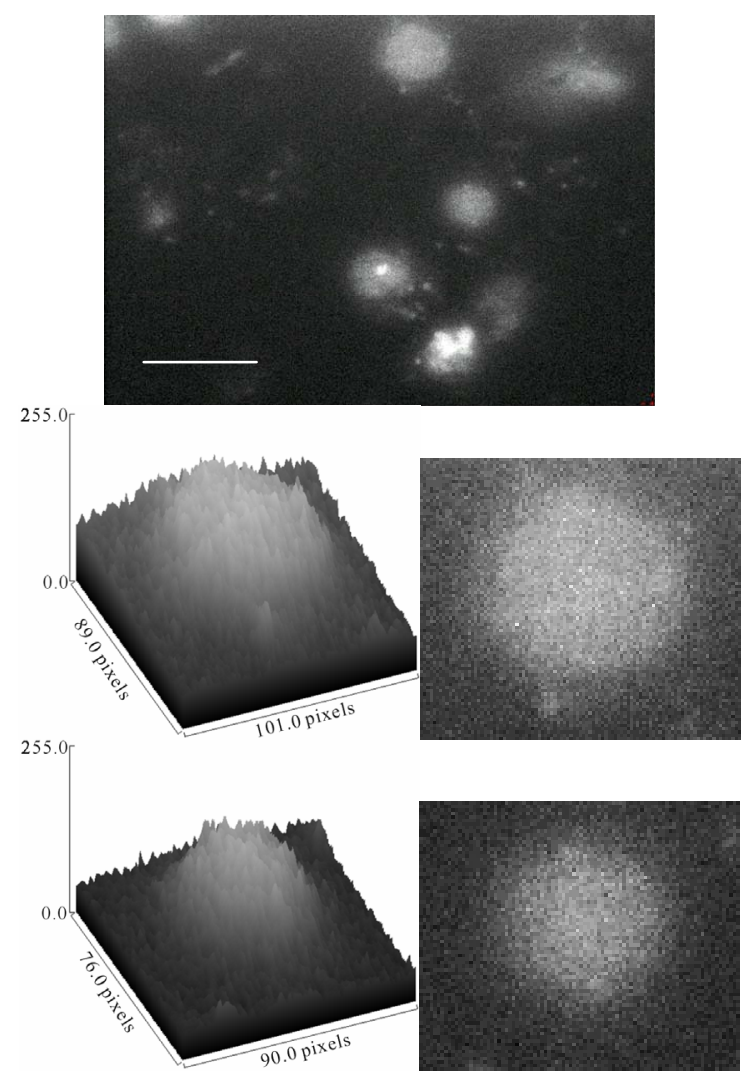

(b)

Figure 3. Cells after LUVs electrofusion observed under fluorescence microscopy. (a) 10 pulses were applied. A general view at moderate magnification, a zoomed view of a single cell and its "surface plot" analysis are given; (b) 20 pulses were applied. A general view at moderate magnification, a zoomed view of two "single" cells and their "surface plot" analysis are given. Cells were observed 4 hours after PEF delivery. The bar is $20 \mu \mathrm{m}$ long.
Is similar to what is observed in the background signal detected outside of the cell. This may nevertheless suggest that some endocytotic vesicles are present. The general view at moderate magnification obtained on cells treated by 20 pulses (Figure 3(b)) shows "hot" spots very clearly (cells in the lower part of the figure). However, this observation is done with a wide field microscope and the intensity of these "hot" spots is very high (saturation of the camera) and so they may correspond to stuck unfused-LUVs on the cell surface, rather than endocytotic vesicles. It is important to note that the homogenous cytoplasm labeling was observed only after 4 $\mathrm{h}$ incubation at $37^{\circ} \mathrm{C}$ in complete culture medium (nanovesicles being washed out). No content mixing was observed if cells were kept at $4^{\circ} \mathrm{C}$ after PEF delivery. Furthermore, more fluorescent cells in the pulsed population are detected when increasing the number of pulses (compared Figures 3(a) and 3(b)). There was however a limit due to the loss of viability even if a protective effect of the nanovesicles was present. Homogeneous FD entry meant that the macromolecules were able to penetrate within the nucleus. This organelle is rather large (see Figure 2 the contrast picture to see its size) and should give a poorly fluorescent area in the fluorescence image if the macromolecules were not able to penetrate. This is indeed not the case as displayed in Figure 3.

\section{Conclusions}

Fusion was obtained by using the electrical parameters previously described to induce the cytoplasmic transfer of the small sized Pyranin [5]. The homogeneity of the cytoplasm fluorescence is due to its content mixing with the internal volume of liposomes. Content mixing with large molecules is the evidence of fusion of the nanovesicles, The process is a content and "membrane" mixing, following neither a "kiss and run" nor an endocytotic pathway [16-18]. The increase in fusion with the number of pulses is linked to an increased fusogenicity with an increase in electrically induced membrane defects. This has been already observed in the fusion of cells with Pyranin loaded vesicles [5] or in cell homofusion [19]. Content mixing for large molecules labeled $20 \mathrm{kD}$ dextrans is obtained only after a long incubation suggesting that a membrane-cytoskeleton reorganization must take place.

Delivery by lipidic nanovesicles is associated to a targeting and avoids the free circulation of the drug. The lack of a direct electrotransfer of labeled $20 \mathrm{kD}$ dextrans was already reported [15]. LUV electrofusion can be targeted either by a biological method (immunoliposomes) or simply by a targeted field delivery through a proper localization of the electrodes.

Electrically enhanced binding of liposomes to cells 
was reported 20 years ago [20]. The spontaneous destabilization of the lipid bilayer of LUVs remains to be explained [21]. The field pulse induces a membrane destabilization which is prone to a spontaneous fusion as proposed by Rosenheck. Our results may be considered within the framework of elucidating the physical basis of exocytosis. The conclusion is that electropermeabilization (and associated fusion) may be endogenously present as already reported for peptide membrane permeabilization [22]. A key feature of our delivery method is that the transfer is obtained after a very short contact period (10 $\mathrm{min}$ in this report), a major advantage for drug delivery. A final advantage is that the electrical conditions can be used in clinical applications [23].

\section{Acknowledgements}

Supports from the region Midi Pyrénées, ANR Cemirbio, PCV CNRS, ARC and Erasmus programs should be acknowledged.

\section{REFERENCES}

[1] E. Neumann, G. Gerisch and K. Optaz, "Cell Fusion Induced by High Electric Impulse Applied to Dictyostelium," Naturwissenschaften, Vol. 67, 1980, pp. 414-415. doi:10.1007/BF00405493

[2] J. Teissie, V. P. Knutson, T. Y. Tsong and M. D. Lane, "Electric Pulse-Induced Fusion of 3T3 Cells in Monolayer Culture," Science, Vol. 216, 1982, pp. 537-538. doi:10.1126/science.7071601

[3] H. Mekid and L. M. Mir, "In Vivo Cell Electrofusion," Biochimica et Biophysica Acta (BBA)—General Subjects, Vol. 1524, No. 2-3, 2000, pp. 118-130. doi:10.1016/S0304-4165(00)00145-8

[4] J. Teissie and M. P. Rols, "Fusion of Mammalian Cells in Culture Is Obtained by Creating the Contact between Cells after Their Electropermeabilization," Biochemical and Biophysical Research Communications, Vol. 140, No. 1, 1986, pp. 258-266. doi:10.1016/0006-291X(86)91084-3

[5] C. Ramos, D. Bonato, M. Winterhalter, T. Stegmann and J. Teissié, "Spontaneous Lipid Vesicle Fusion with Electropermeabilized Cells," FEBS Letters, Vol. 518, 2002, pp. 135-138. doi:10.1016/S0014-5793(02)02676-5

[6] J. Heuving, F. Pincet and S. Cribier, "Hemifusion and Fusion of Giant Vesicles Induced by Reduction of Inter-Membrane Distance," The European Physical Journal E: Soft Matter and Biological Physics, Vol. 14, No. 3, 2004, pp. 269-276. doi:10.1140/epje/i2003-10151-2

[7] D. Tareste, F. Pincet, E. Perez, S. Rickling, C. Mioskowski and L. Lebeau, "Energy of Hydrogen Bonds Probed by the Adhesion of Functionalized Lipid Layers," Biophysical Journal, Vol. 83, No. 6, 2002, pp. 3675-3681. doi:10.1016/S0006-3495(02)75367-8

[8] F. Pincet, L. Lebeau and S. Cribier, "Short-Range Specific Forces Are Able to Induce Hemifusion," European
Biophysics Journal, Vol. 30, No. 2, 2001, pp. 91-97. doi:10.1007/s002490100131

[9] C. G. Schuette, K. Hatsuzawa, M. Margittai, A. Stein, D. Riedel, P. Küster, M. König, C. Seidel and R. Jahn, “Determinants of Liposome Fusion Mediated by Synaptic SNARE Proteins," Proceedings of the National Academy of Sciences USA, Vol. 101, No. 9, 2004, pp. 2858-2863. doi:10.1073/pnas.0400044101

[10] A. Cypionka, A. Stein, J. M. Hernandez, H. Hippchen, R. Jahn and P. J. Walla, "Discrimination between Docking and Fusion of Liposomes Reconstituted with Neuronal Snare-Proteins Using FCS," Proceedings of the National Academy of Sciences USA, Vol. 106, No. 44, 2009, pp. 18575-18580. doi:10.1073/pnas.0906677106

[11] W. Xu and F. Pincet, "Quantification of Phase Transitions of Lipid Mixtures from Bilayer to Non-Bilayer Structures: Model, Experimental Validation and Implication on Membrane Fusion," Chemistry and Physics of Lipids, Vol. 163, No. 3, 2010, pp. 280-285. doi:10.1016/j.chemphyslip.2009.12.002

[12] S. Martens, M. M. Kozlov and H. T. McMahon, "How Synaptotagmin Promotes Membrane Fusion," Science, Vol. 316, No. 5828, 2007, pp. 1205-1208. doi:10.1126/science. 1142614

[13] K. Rosenheck, "Evaluation of the Electrostatic Field Strength at the Site of Exocytosis in Adrenal Chromaffin Cells," Biophysical Journal, Vol. 75, 1998, pp. 12374123. doi:10.1016/S0006-3495(98)74043-3

[14] P. Luitel, D. F. Schroeter and J. W. Powell, "Self-Electroporation as a Model for Fusion Pore Formation," Journal of Biomolecular Structure \& Dynamics, Vol. 24, No. 5, 2007, pp. 495-503.

[15] M. P. Rols and J. Teissie, "Electropermeabilization of Mammalian Cells to Macromolecules: Control by Pulse Duration," Biophysical Journal, Vol. 75, No. 3, 1998, pp. 1415-1423. doi:10.1016/S0006-3495(98)74060-3

[16] E. R. Travis and R. M. Wightman, "Spatio-Temporal Resolution of Exocytosis from Individual Cells," Annual Review of Biophysics and Biomolecular Structure, Vol. 27, 1998, pp. 77-103. doi:10.1146/annurev.biophys.27.1.77

[17] E. Alés, L. Tabares, J. M. Poyato, V. Valero, M. Lindau and G. Alvarez de Toledo, "High Calcium Concentrations Shift the Mode of Exocytosis to the Kiss-and-Run Mechanism," Nature Cell Biology, Vol. 1, No. 1, 1999, pp. 40-44. doi:10.1038/9012

[18] S. O. Rizzoli and R. Jahn, "Kiss-and-Run, Collapse and 'Readily Retrievable' Vesicles," Traffic, Vol. 8, No. 9, 2007, pp. 1137-1144. doi:10.1111/j.1600-0854.2007.00614.x

[19] J. Teissie and C. Ramos, "Correlation between Electric Field Pulse Induced Long-Lived Permeabilization and Fusogenicity in Cell Membranes," Biophysical Journal, Vol. 74, 1998, pp. 1889-1898. doi:10.1016/S0006-3495(98)77898-1

[20] L. V. Chernomordik, D. Papahadjopoulos and T. Y. Tsong, "Increased Binding of Liposomes to Cells by 
Electric Treatment," Biochimica et Biophysica Acta $(B B A)$-General Subjects, Vol. 1070, No. 1, 1991, pp. 193-197. doi:10.1016/0005-2736(91)90163-3

[21] J. Teissie, M. Golzio and M. P. Rols, "Mechanisms of Cell Membrane Electropermeabilization: A Minireview of Our Present (Lack of ?) Knowledge," Biochimica et Biophysica Acta (BBA)-General Subjects, Vol. 1724, No. 3, 2005, pp. 270-280. doi:10.1016/j.bbagen.2005.05.006

[22] M. Miteva, M. Andersson, A. Karshikoff and G. Otting,
"Molecular Electroporation: A Unifying Concept for the Description of Membrane Pore Formation by Antibacterial Peptides, Exemplified with NK-lysin," FEBS Letters, Vol. 462, No. 1-2, 1999, pp. 155-158. doi:10.1016/S0014-5793(99)01520-3

[23] T. Hampton, "Electric Pulses Help with Chemotherapy may Open New Paths for Other Agents," Journal of the American Medical Association (JAMA), Vol. 305, No. 6, 2011, pp. 549-551. doi:10.1001/jama.2011.92 